\title{
MicroRNA-let-7a regulates cell autophagy by targeting Rictor in gastric cancer cell lines MGC-803 and SGC-7901
}

\author{
HAO FAN ${ }^{1 *}$, MINGKUN JIANG $^{1 *}$, BOWEN LI $^{1 *}$, YU HE $^{1}, \mathrm{CHI} \mathrm{HUANG}^{1}$, \\ DAKUI LUO $^{1}$, HAO XU ${ }^{1}$, LI YANG $^{1}$ and JUNDONG ZHOU ${ }^{2}$ \\ ${ }^{1}$ Department of General Surgery, The First Affiliated Hospital of Nanjing Medical University, Nanjing, Jiangsu 210029; \\ ${ }^{2}$ The Core Laboratory of The Suzhou Cancer Center and Department of Radiotherapy, \\ Nanjing Medical University Affiliated Suzhou Hospital, \\ Suzhou, Jiangsu 215001, P.R. China
}

Received May 27, 2017; Accepted December 29, 2017

DOI: $10.3892 /$ or.2018.6194

\begin{abstract}
R-let-7a is the most widely studied miRNA, whose functions have been well-established by scientists in both carcinogenesis and progression of human cancer, including gastric cancer (GC). However, to date there is a lack of information concerning the relationship between miR-let-7a and cellular autophagy. Using western blotting and immunofluorescence, we determined that upregulation of miR-let-7a led to increased cellular autophagic level, whereas miR-let-7a suppression decreased autophagy activity in GC cells. To further elucidate the mechanisms underlying this, we screened potential targets of miR-let-7a using bioinformatics analyses, validated by a series of assays. Our results indicated that Rptor independent companion of mTOR complex 2 (Rictor) was a direct target of miR-let-7a. In addition, rescue experiments in vitro showed that miR-let-7a promoted cellular autophagic level by inhibiting Rictor expression in GC cells. Furthermore, as an upstream executor of Akt-mTOR signaling pathway, we found that Rictor elaborated its effect on autophagy by phosphorylating Akt and mTOR, and this regulatory process could also be mediated by miR-let-7a. Taken together, our results present a novel role for miR-let-7a in GC which modulates
\end{abstract}

Correspondence to: Dr Li Yang, Department of General Surgery, The First Affiliated Hospital of Nanjing Medical University, 300 Guangzhou Road, Nanjing, Jiangsu 210029, P.R. China

E-mail:pwkyangli@163.com

Dr Jundong Zhou, The Core Laboratory of The Suzhou Cancer Center and Department of Radiotherapy, Nanjing Medical University Affiliated Suzhou Hospital, 16 West Baita Road, Suzhou, Jiangsu 215001, P.R. China

E-mail: zhoujundong330@163.com

*Contributed equally

Key words: gastric cancer, autophagy, miR-let-7a, Rictor, mTOR autophagy by targeting Rictor, following the regulation of Akt-mTOR signal pathway.

\section{Introduction}

Gastric cancer (GC) is one of the primary causes of tumorassociated deaths worldwide, most cases occur in East Asia, and the mortality rates are high (1). In China, GC represents the third most established malignancy (2). The incidence of GC has decreased with the improved health conditions and the optimized medical level. However, due to the high rates of metastasis and recurrence, the 5-year overall survival rate of advanced GC is $20 \%$ (3). New therapeutic strategies such as targeting molecules have been reported in GC (4). Hence, the study of new treatments that manage this disease is urgent.

Autophagy is an evolutionarily conserved process that includes the degradation of intracellular organelles and cytoplasmic components by lysosomes (5), this process is effective in maintaining cellular homeostasis $(6,7)$. However, the effects of autophagy on particular cellular functions are sometimes contradictory, and autophagy is involved in the pathway of cell survival and cell death under metabolic stress $(8,9)$. It has been reported that autophagy exerted influence on tumorigenesis and therapy $(10,11)$.

MicroRNAs (miRNAs) are a class of endogenous, highly conserved non-coding small RNA, which could bind to the 3'-untranslated region (3'-UTR) of the target gene and restrain translation or accelerate their degradation, thereby regulating the expression of the target gene at post-transcriptional or translation level $(12,13)$. It has been reported that miRNAs are implicated in regulating autophagy in a variety of diseases (14-16). Our previous study demonstrated that miR-let7a functions as antitumor gene and inhibits cell proliferation, migration and invasion in GC cells (17).

Rictor (Rptor independent companion of mTOR complex 2) is a core component of mTORC2, and mTORC 2 is one part of mTOR (the mechanistic target of rapamycin) signaling pathway, mTOR signaling pathway is related to regulation of cell autophagy $(18,19)$. Rictor has also been reported to be involved in autophagy process (20). Rictor was predicted as the target protein of miR-let-7a through exploiting bioinformatics 
software including TargetScan and miRanda, and was verified by dual-luciferase reporter assay (21-23).

In the present study, we revealed the impact of miR-let-7a on Rictor expression and its molecular mechanism in the regulation of autophagy in GC cell. Firstly, we found that overexpression of miR-let-7a increased autophagic activity, while knockdown had the reverse effect. Next, we found that Rictor was the direct functional target of miR-let-7a. Finally, miR-let-7a promoted autophagic activity by suppressing the activation of Akt-mTOR pathway.

\section{Materials and methods}

Culture of human GC cells. Human GC cell lines MGC-803, SGC-7901, BGC-823, AGS and human normal gastric mucous epithelium cell GES-1 were obtained from the American Type Culture Collection (ATCC; Manassas, VA, USA), and were grown in RPMI-1640 medium, supplemented with $10 \%$ fetal bovine serum (FBS) and 1\% antibiotics (penicillinstreptomycin) (all from Gibco, Carlsbad, CA, USA). All cells were cultured in humidified incubator containing $5 \% \mathrm{CO}_{2}$ at $37^{\circ} \mathrm{C}$.

Quantitative real-time PCR analysis for $m R N A$ and miRNA. Total RNA were isolated from cells using TRIzol reagent (Invitrogen, Carlsbad, CA, USA) according to the manufacturer's instructions. TaqMan miRNA assays were performed to determine the expression of miR-let-7a. TaqMan miRNA reverse transcription kit (Invitrogen) was used for cDNA synthesis and qRT-PCR was carried out by ABI StepOne Plus (Applied Biosystems, Foster City, CA, USA). U6 was an endogenous reference for miR-let-7a quantification using the $2^{-\Delta \Delta \mathrm{Ct}}$ method. The relative expression of Rictor was quantifed using SYBR-Green Master Mix kit (Roche Diagnostics, Indianapolis, IN, USA) and $\beta$-actin was selected as control. The specific primers were as follows: miR-let-7a forward, 5'-GGTGAGGTAGTAGGTTGTATAGTT-3' and reverse, 5'-CTCGCTTCGGCAGCACATATA-3'; U6 forward, 5'-CTC GCTTCGGCAGCACA-3' and reverse, 5'-AACGCTTCACGA ATTTGCGT-3'; Rictor forward, 5'-ACCGGGCTTCTGACCA TTAAA-3' and reverse, 5'TTGTATGAACCGCCGACACT-3'; $\beta$-actin forward, 5'-AGAAAATCTGGCACCACACC-3' and reverse, 5'-TAGCACAGCCTGGATAGCAA-3'. All data collected were in triplicate.

Western blot analysis. RIPA buffer (Beyotime, Shanghai, China) was used to isolate total proteins from cell lysates on ice. Equal amounts of protein were separated by $10 \%$ SDS polyacrylamide gel electrophoresis. Proteins on the gel were transferred to polyvinylidene difluoride (PVDF) membranes. Then, the membranes were blocked with Tris-buffered saline with Tween-20 (TBST) containing 5\% milk for $2 \mathrm{~h}$ and incubated overnight in special primary antibodies: anti-LC3, anti-p62, anti-Rictor, anti-Akt, anti-p-Akt, anti-mTOR, anti-pmTOR and anti-GAPDH (dilution 1:1,000; Cell Signaling Technology, Beverly, MA, USA). After being washed with TBST three times 15-min each, the HRR-conjugated antimouse or anti-rabbit IgG antibodies (dilution 1:20,000; Jackson ImmunoResearch, Inc., West Grove, PA, USA) were incubated with bolted membranes at room temperature for $2 \mathrm{~h}$ and washed again. The enhanced chemiluminescence detection system was used to detect the expression of target bands.

Immunofluorescence. Cells were seeded into glass bottom cell culture dish at 20,000 cells and cultured for $48 \mathrm{~h}$. After being washed with PBS three times, cells were fixed in $4 \%$ paraformaldehyde for $20 \mathrm{~min}$ and permeabilized by $0.5 \%$ Triton X-100 for $10 \mathrm{~min}$. Then, cells were blocked with $3 \%$ bovine serum albumin for $1 \mathrm{~h}$ and incubated overnight in special primary antibody: anti-LC3 (dilution 1:200). After removing primary antibody and washing again with PBS next day, cells were incubated with Alexa Flour 594 Rhodamine-conjugated goat anti-rabbit (dilution 1:200; Jackson ImmunoResearch) for $1 \mathrm{~h}$ and $30 \mathrm{~min}$ and mounted by staining with 4',6-diamidino2-phenylindole (DAPI) for $5 \mathrm{~min}$ at room temperature. Confocal microscopy (Carl Zeiss LSM710; Carl Zeiss, Jena, Germany) was used to photograph the stained cells in a $\mathrm{x} 40$ oil lens and LC3 puncta was manually quantified.

Cell transfection. The miR-let-7a mimics and miR-let-7a inhibitor vector lentiviral constructs (GenePharma, Shanghai, China) were modified to interfere with expression of miR-let7a, as were siRictor and pcDNA3.1-Rictor to interfere with expression of Rictor from Santa Cruz Biotechnology (Dallas, TX, USA). Negative miRNA-let-7a and scramble Rictor were also transfected as negative controls. When MGC-803, SGC-7901 and GES-1 cells grew to 50-60\% confluency, the miR-let-7a-NC, miR-let-7a mimics and miR-let-7a inhibitor lentiviral vectors were used to infect cells according to different multiplicity of infection (MOI) offered by the manufacturer. Puromycin ( $3 \mu \mathrm{g} / \mathrm{ml})$ (Sigma, St. Louis, MO, USA) was used to screen out stable cell lines for one week and the expression of miR-let-7a was analyzed by qRT-PCR. siRNA against Rictor and pcDNA3.1-Rictor plasmids were transfected into GC cells using Lipofectamine 3000 reagent (Invitrogen).

Dual-luciferase reporter assay. The wild-type (WT) or mutated-type (MUT) 3'-UTRs of Rictor containing miR-let-7a binding sites were amplified by PCR and subcloned into pmirGLO Dual-Luciferase miRNA Target Expression vectors (Promega, Madison, WI, USA). For luciferase activity assays, HEK293T cells were seeded into a 24-well plate, then co-transfected with Rictor 3'-UTR construct and miR-let-7a or NC using Lipofectamine 3000 (Invitrogen). Luciferase activity was measured by Dual-Luciferase Reporter assay kit (Promega, USA) and normalized by Renilla luciferase overnight.

Statistical analysis. Data are presented as mean \pm standard deviation (SD) from at least three independent experiments and the Student's two-tailed t-test was used to determine whether these data are statistically significant. Values of $\mathrm{P}<0.05$ were considered significant.

\section{Results}

miR-let-7a affects autophagic activity in GC cells. To elucidate the role of miR-let-7a on autophagic activity in GC, we firstly constructed miR-let-7a mimics and miR-let-7a inhibitors using lentiviral transfection in GC cells (MGC-803 and SGC-7901). miRNA real-time polymerase chain reaction (RT-PCR) was 


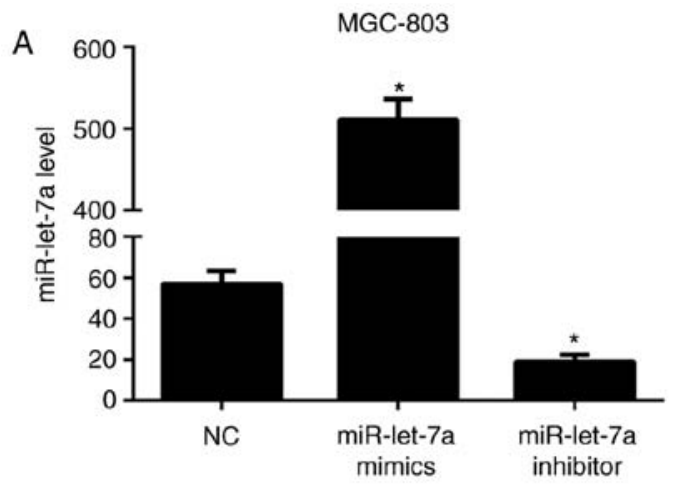

c

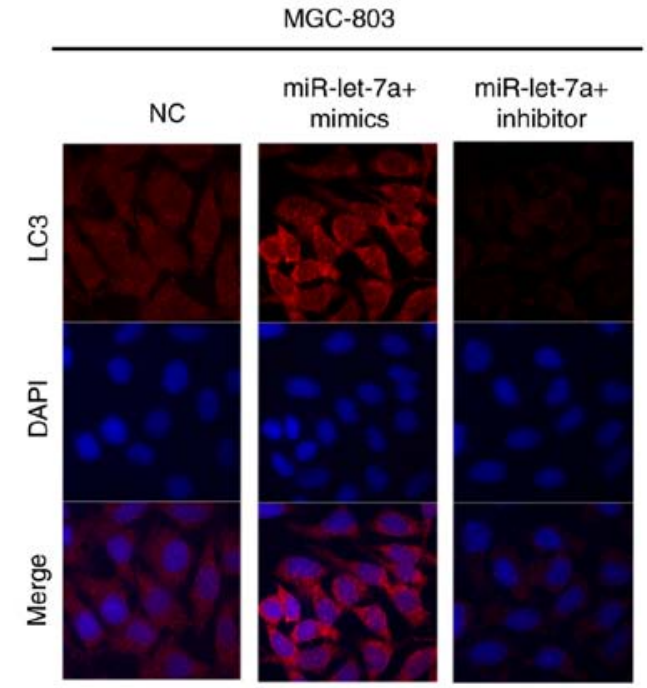

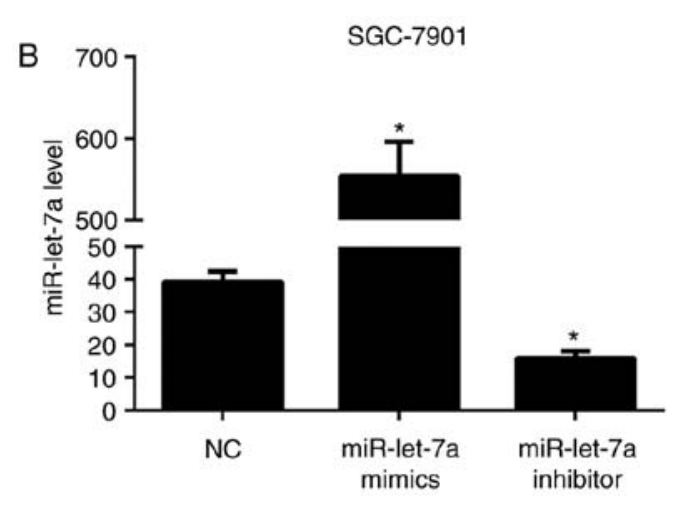

D

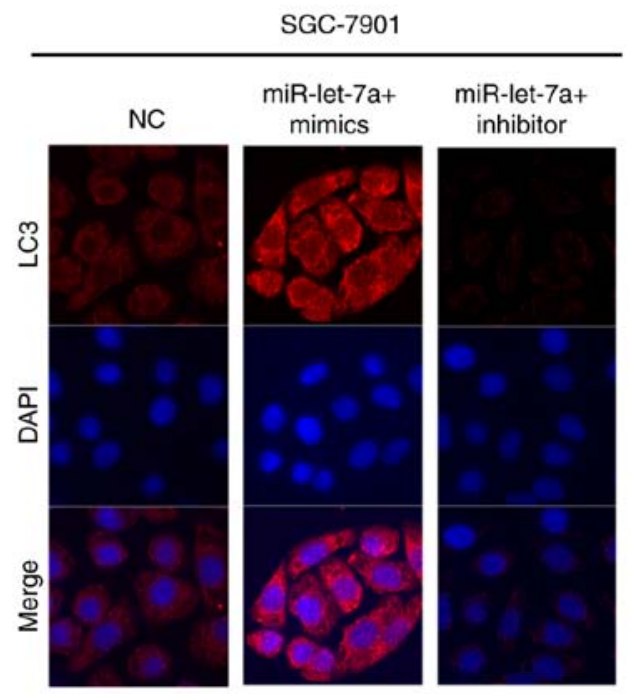

Figure 1. miR-let-7a affects autophagic activity in gastric cancer cells. (A and B) MGC-803 and SGC-7901 cells were transfected with miR-let-7a mimics, miR-let-7a inhibitor, empty lentiviral construct vectors as negative control (NC), respectively. Cells were collected for miRNA RT-PCR to quantify the expression levels of miR-let-7a. Independent experiments were performed in triplicate; ${ }^{*} \mathrm{P}<0.05$ compared with NC. (C and D) Immuofluorescence staining of LC3 puncta were visualized by confocal imaging in MGC-803 and SGC-7901 cells after transfection. Red represents LC3 staining, blue stands for nuclear DNA staining by DAPI (magnification, $\mathrm{x} 40$ ).

used to determine the expression of miR-let-7a (Fig. 1A and B). As shown, miR-let-7a was knocked downed $\sim 65$ and $60 \%$, increased appoximately 9- and 14-fold in MGC-803 and SGC-7901 cells, respectively. Next, we explored the effect of miR-let-7a on autophagy, confocal imaging was employed to evaluate the number of LC3 puncta (Fig. 1C and D). It showed that the number of LC3 puncta were markedly elevated in MGC-803 and SGC-7901 cells which were overexpressed of miR-let-7a. However, the LC3 dot formation was significantly reduced after knocking down miR-let-7a both in MGC-803 and SGC-7901 cells. These results recognized miR-let-7a as an enhancer of autophagy and it contributes to the promotion of autophagic responses in GC cells.

miR-let-7a affects autophagic flux in GC cells. The conversion of LC3-I to LC3-II and p62 protein expression were detected by western blotting. LC3-I scattered in the cytoplasm, and upon autophagy induction, LC3-I was conjugated with phosphatidylethanolamine (PE) to form LC3-II and localized to autophagosomes and adventitia. LC3-II is always retained on the autophagic membrane until it is fused to lysosomes. We found that LC3-II/GAPDH ratios were increased after overexpressing miR-let-7a and were decreased after downregulating miR-let-7a, these results were consistent with previous LC3 puncta formation assay (Fig. 2A and B, and D and E). P62 is a selective autophagic-lysosomal degradation substrate, total cellular p62 protein levels reflect autophagic activity. When autophagy occurs, p62 levels are decreased, while autophagic activity is inhibited, p62 protein is accumulated. As expected, overexpression of miR-let-7a led to reduced p62 protein levels, but inhibition of miR-let-7a resulted in an increase of p62 expression (Fig. 2A, C, D and F). Then, we performed an autophagic flux assay to determine whether autophagosome accumulation resulted from increased autophagic activity or due to a block in downstream degradation. Chloroquine (CQ), a lysosomotropic reagent, was used to block autophagosome degradation in MGC-803 and SGC-7901 cells. As shown, lipidated LC3-II was significantly increased both in NC and miR-let-7a transfected cells by CQ (Fig. 2A and B and D and E). Similarly, p62 protein levels were also increased in miR-let-7a transfected cells treated with CQ (Fig. 2A, C, D and F). These results demonstrate that miR-let-7a affected autophagic activity in GC cells.

miR-let-7a directly binds to the 3' UTR of Rictor. In order to clarify the specific mechanism of miR-let-7a-induced autophagy, bioinformatics software including TargetScan and miRanda were employed to predict the potential binding 
A
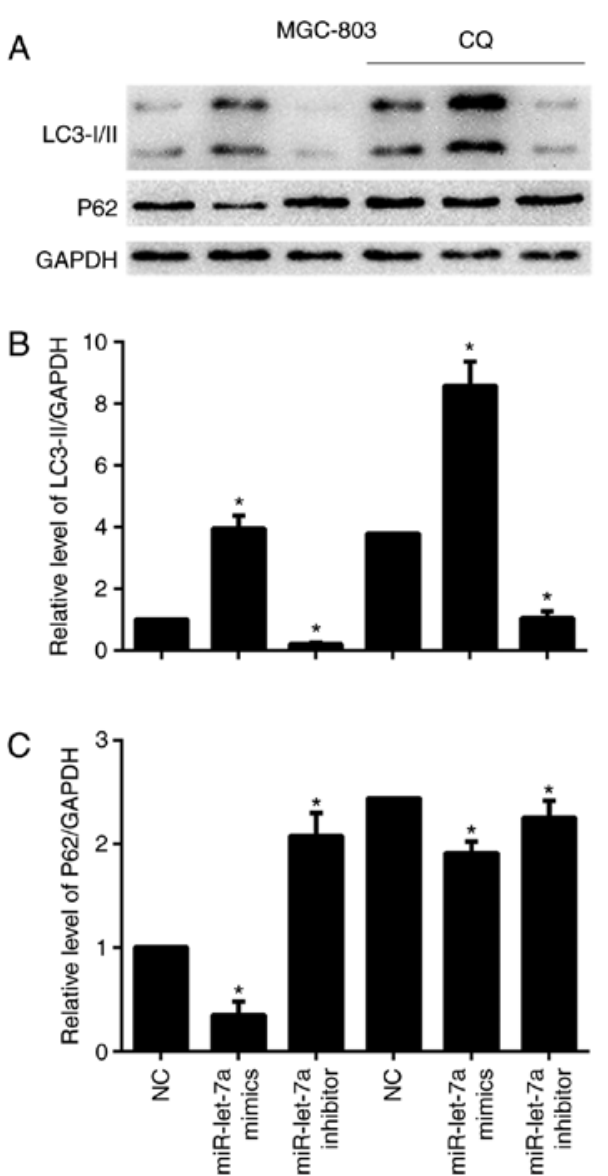

D

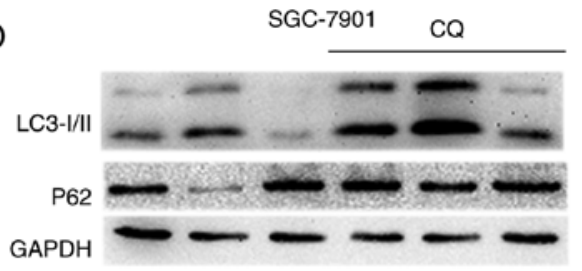

E
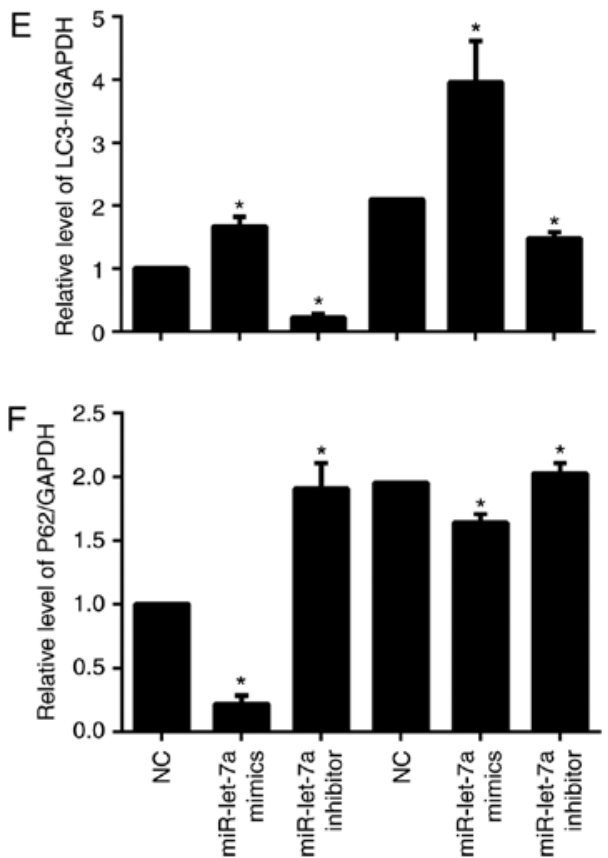

Figure 2. miR-let-7a affects autophagic flux in gastric cancer cells. (A and D) Western blot analysis of the expression level of LC3 conversion and p62 after GC cells were transfected with miR-let-7a mimics, miR-let-7a inhibitor and NC, respectively. Then, MGC-803 and SGC-7901 cells were treated with chloroquine for $24 \mathrm{~h}$ and harvested for western blotting. (B and C, and E and F) ImageJ densitometric analysis were used to detect the LC3-II/GAPDH or p62/GAPDH ratios from western blotting. Relative expression values are represented as mean \pm SD from three independent experiments; ${ }^{2}<0.05$ compared with $\mathrm{NC}$.

sequences in the 3'-untranslated region (3'-UTR) of target genes. As shown, the 3'-UTR of human Rictor mRNA resides underlying miR-let-7a binding sequences (Fig. 3A). Furthermore, the wild-type (WT) or mutated-type (MUT) sequences in the 3'-UTR of Rictor that miR-let-7a targets were designed to construct luciferase reporter vector and co-transfected with either miR-let-7a or NC control. As shown, overexpression of miR-let-7a markedly reduced luciferase activity when HEK293T cells were co-transfected with WT 3'-UTR of Rictor compared to NC. In contrast, this inhibitory effect of miR-let-7a on luciferase activity was abrogated when HEK293T cells were co-transfected with MUT (Fig. 3B).

We performed RT-PCR assay and western blot analysis to further confirm whether miR-let-7a suppressed the expression of Rictor in GC cells. As shown, overexpression of miR-let-7a led to a significant decrease both in mRNA and protein levels in MGC-803 and SGC-7901 cells compared with NC. However, inhibition of miR-let-7a notably increased the levels of Rictor mRNA and protein (Fig. 3C-H). Therefore, the data above provided strong evidence that Rictor is the target of miR-let-7a, and miR-let-7a inhibits the expression of Rictor by directly binding to its 3'-UTR.

Rictor reverses autophagic activity regulated by miR-let-7a. To explore whether the effect of miR-let-7a on GC cell autophagic activity was mediated by Rictor, we performed 'rescue experiments'. GC cell lines MGC-803 and SGC-7901 were co-transfected with miR-let-7a overexpression lentivirus and either pcDNA3.1-Rictor plasmids or empty vector, worthwhile, other MGC-803 and SGC-7901 cells were co-transfected with miR-let-7a supression lentivirus and either siRictor or empty vector, respectively. As shown, upregulated Rictor expression reversed the promotion of autophagic activity caused by the overexpression of miR-let-7a, the results suggested that LC3-II/GAPDH ratios were decreased and p62 protein levels were increased in MGC-803 and SGC-7901 cells (Fig. 4A and B). Simultaneously, we found that the similar rescue effect was observed in MGC-803 and SGC-7901 cells where downregulated Rictor expression reversed the supression of autophagic activity caused by the knockdown of miR-let-7a (Fig. 4A and B).

Rapamycin, as a potent immunosuppressive and antitumor agent (24), is commonly used in autophagy study. As a control, we found that miR-let-7a enhanced the activity of autophagy as well as rapamycin by confocal imaging in MGC-803 cells, and LC3 dots formation was reversed upon co-expression with Rictor (Fig. 4C). Inhibition of endogenous miR-let-7a led to the opposite effect (Fig. 4D). These results show that Rictor is the vital functional element in miR-let-7a-mediated autophagic response. 
A Rictor 3' UTR WT: 5' AUUCAUUUUAAACACUACCUCA 3' miR-let-7a: 3' UUGAUAUGUUGGAUGAUGGAGU 5' Rictor 3' UTR MUT : 5' AUUCAUUUUAAACGCGAUAUUA $3^{\prime}$

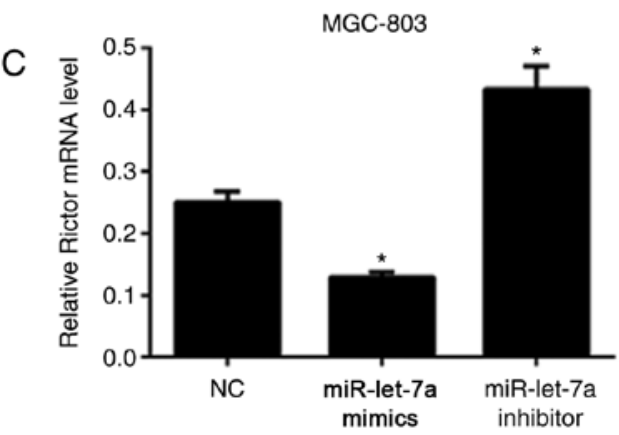

E MGC-803

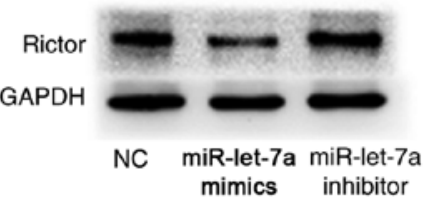

G

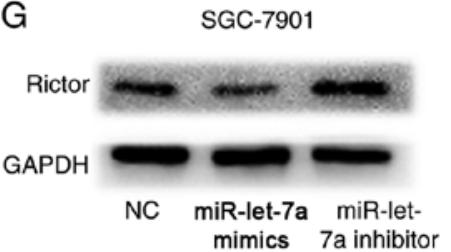

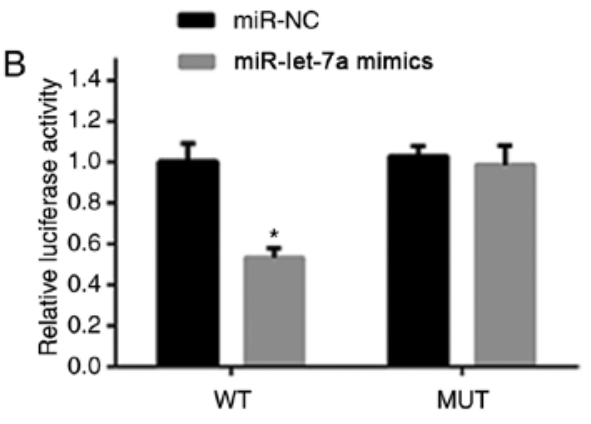
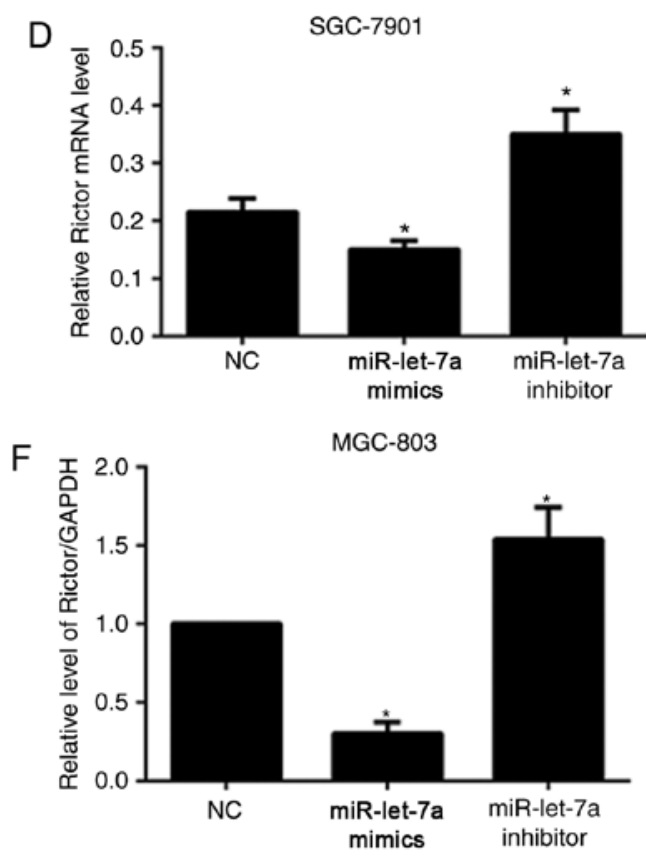

$\mathrm{H}$

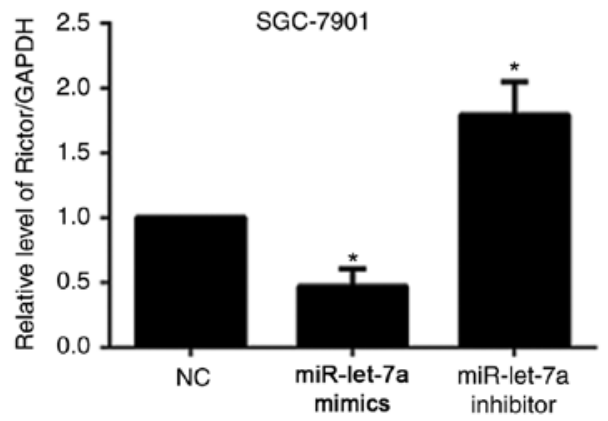

Figure 3. Rictor is a direct target of miR-let-7a in gastric cancer cells. (A) The predicted binding sequence from miR-let-7a and targeting sites from the 3'-UTR of Rictor. (B) Luciferase reporter constructs containing either wild-type (WT) or mutated (MUT) Rictor 3'-UTR were co-transfected with miR-let-7a mimics or NC. Luciferase activity was evaluated at $48 \mathrm{~h}$ after transfection and normalized relative to the Renilla luciferase expression. Data are presented as the mean \pm SD from three independent experiments; ${ }^{*} \mathrm{P}<0.05$ compared with NC. (C and D) The mRNA levels of Rictor were analyzed by qRT-PCR in MGC-803 and SGC-7901 cells after transfection; ${ }^{*} \mathrm{P}<0.05$ compared with NC. (E and G) Western blot analysis was performed to detect the expression of Rictor after upregulation or downregulation of miR-let-7a in GC cells. (F and H) Relative densities of Rictor from western blotting were quantified using ImageJ software. All the experiments were performed in triplicate; $\mathrm{P}<0.05$ compared with $\mathrm{NC}$.

miR-let-7a regulates the Akt-mTOR signaling pathway. It has been reported that Akt-mTOR signaling pathway is involved in autophagic activity and Rictor plays an important role in the Akt-mTOR signaling pathway (21). We confirmed that Akt and mTOR phosphorylations were involved in miR-let-7a-mediated autophagy in GC cells by western blot analysis. As shown, both the protein levels of phosphorylated Akt and phosphorylated mTOR were significantly decreased by upregulating miR-let$7 \mathrm{a}$ and increased by downregulating miR-let-7a in MGC-803 and SGC-7901 cells. However, the total protein levels were almost unchanged (Fig. 5A and B). These data indicated that there is a direct link between miR-let-7a and Akt-mTOR signaling pathway. Therefore, we confirm that miR-let-7a regulates autophagic activity throughout the Akt-mTOR signaling pathway in GC cells.

Knockdown of miR-let-7a suppresses human normal gastric mucous epithelium cell autophagic activity. To investigate whether miR-let-7a affects autophagy in human normal gastric mucous epithelium cells (GES-1), we detected the expression 
A

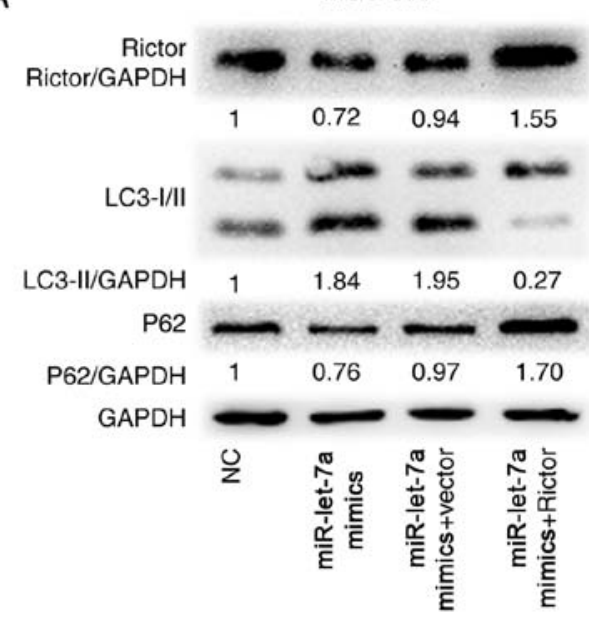

C

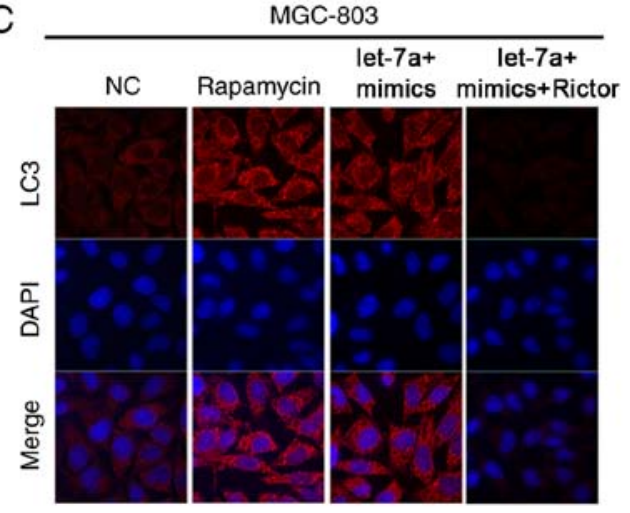

B

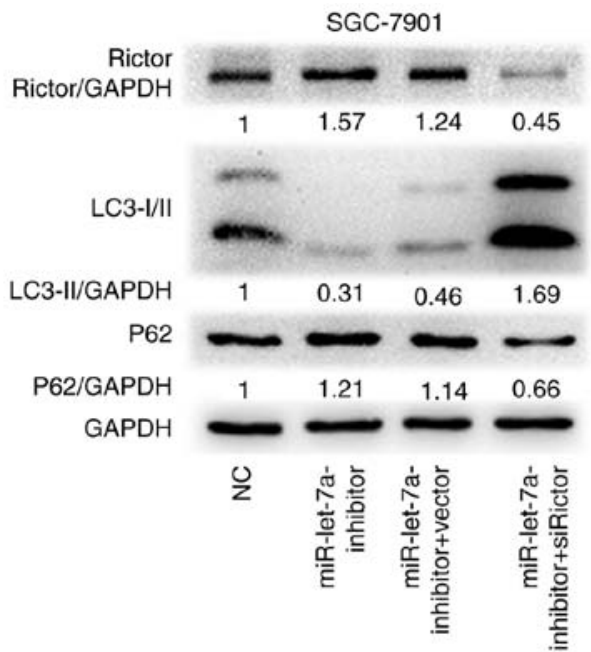

D

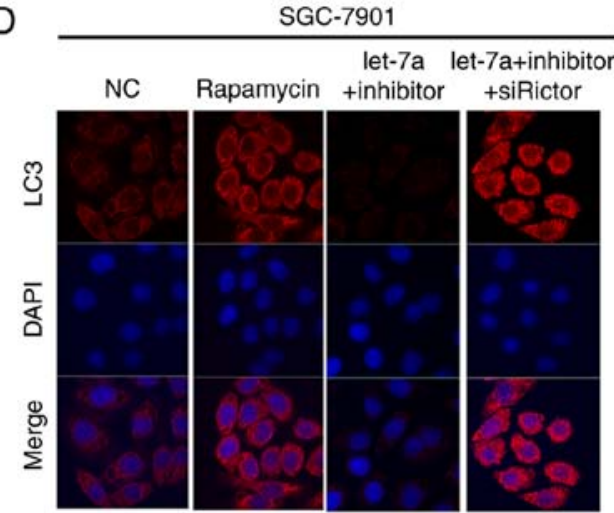

Figure 4. Rictor reverses autophagic activity from miR-let-7a mediated autophagy regulation in gastric cancer cells. (A and B) Western blotting showed that cells treated with Rictor plasmid decreased the levels of LC3 and p62 caused by upregulation of miR-let-7a, inhibition of autophagic activity caused by downregulation miR-let-7a was counteracted by downregulation of Rictor. (C and D) Results from confocal imaging were consistent with that from western blotting in MGC-803 and SGC-7901 cells.

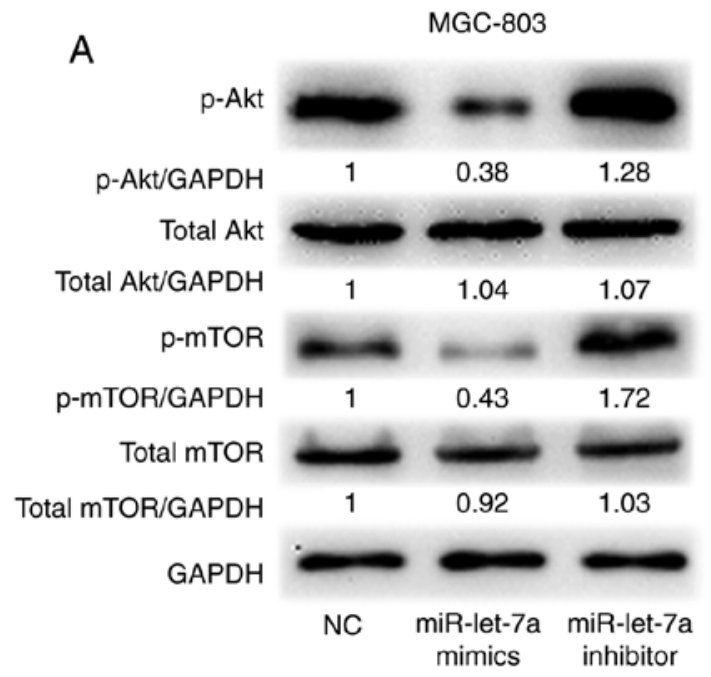

C

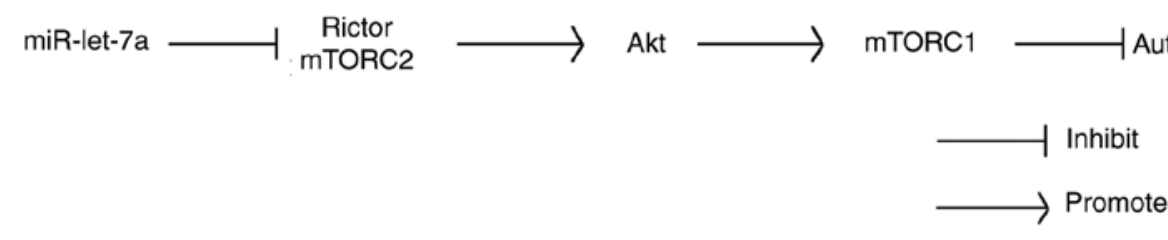

B

SGC-7901

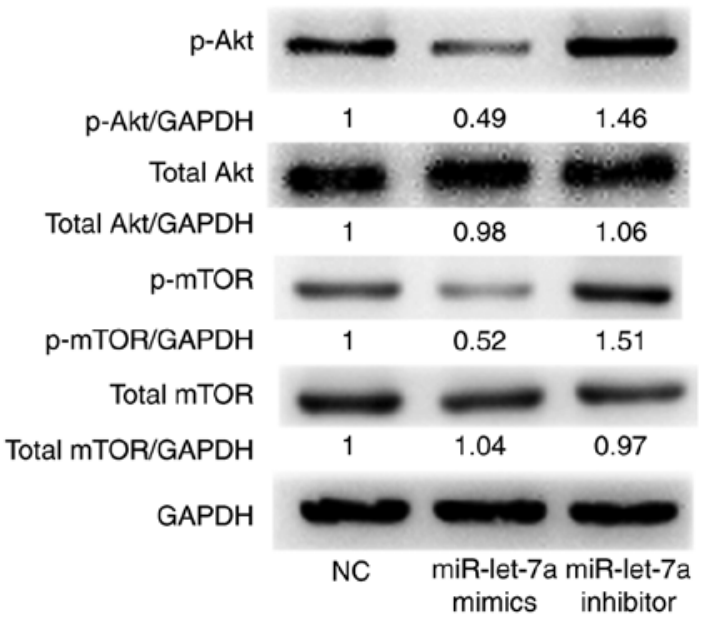

GAPDH NC miR-let-7a miR-let-7a Autophagy

Figure 5. Effects of miR-let-7a on the Akt-mTOR signalling pathway in gastric cancer cells. (A and B) The levels of total mTOR, total Akt, phosphorylated mTOR and phosphorylated Akt were detected by western blotting in MGC-803 and SGC-7901 cells. Protein ratios were obtained following imageJ densitometric analysis. Relative expression values from three independent experiments gave similar results. 

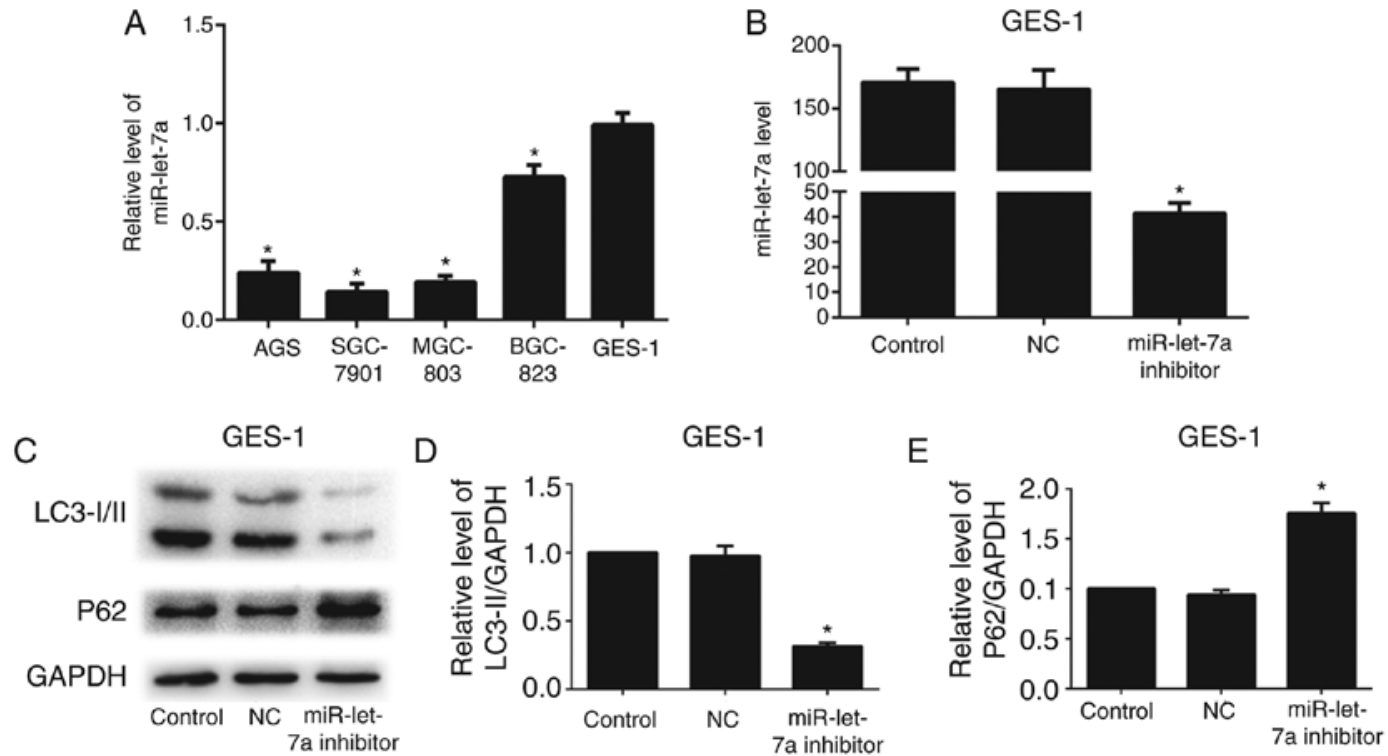

Figure 6. Knockdown of miR-let-7a suppresses human normal gastric mucous epithelium cell autophagic activity. (A) The expression of miR-let-7a were detected by RT-PCR in gastric cancer cell lines and normal gastric mucosa epithelial cell line (GES-1). (B) RT-PCR analysis of miR-let-7a expression levels in GES-1 cells following transfected with miR-let-7a inhibitor or empty lentiviral construct vectors. (C) The expression level of LC3 conversion and p62 were analyzed by western blotting in the miR-let-7a-inhibitor and negative control group. (D and E) Relative densities of Rictor from western blotting were quantified using ImageJ software. All the experiments were performed in triplicate; ${ }^{*} \mathrm{P}<0.05$ compared with $\mathrm{NC}$.

level of miR-let-7a in GC cells and GES-1. We found that the expression of miR-let-7a in GES-1 cells was relatively higher than that in GC cells (Fig. 6A). Thus, we transfected GES-1 cells with miR-let-7a inhibitor or NC. Successful knockdown of miR-let-7a was confirmed by RT-PCR (Fig. 6B). The expression of LC3-II was reduced, and P62 was relatively higher compared with control or NC after knockdown of miR-let-7a by western blotting (Fig. 6C-E). These results suggest that miR-let-7a affects autophagy in human normal gastric mucous epithelium cells.

\section{Discussion}

Accumulating evidence has demonstrated that miRNAs are implicated in modulating autophagic activity through targeting their mRNAs or regulating other signalling pathways (25-27). In our previous study, we reported that miR-let-7a inhibits cell proliferation, migration and invasion in gastric cancer (GC) cells (17). However, it is not clear how miR-let-7a suppresses GC cells by influencing the autophagy pathways. In the present study, we provide insightful evidence to identify miR-let-7a as a powerful enhancer of autophagy in GC cells. Here, we discovered that miR-let-7a promotes autophagy by directly targeting the autophagy-related gene Rictor and that miRlet-7a could directly pair to the 3'-UTR sequence of Rictor, leading to translational repression in GC cells. By suppressing the motivation of Akt-mTOR signalling pathway, miR-let-7a induces autophagic activity and regulates the growth of GC development. Therefore, we introduced miR-let-7a as a potent autophagy inducer which inhibited Rictor-mediated Akt-Mtor activity (Fig. 5C). Combining with previous finding (17), miRlet-7a attributed to autophagy a pro-death role in GC cell lines MGC-803 and SGC-7901. This further optimized the mechanisms of miR-Let7a to serve as a tumor-suppressor in GC, as well as its clinical value.
LC3, microtubule-associated protein 1 light chain 3, is autophagic membrane-labeled protein. There are two forms of LC3 protein in the cell, the LC3-I and LC3-II. The formation of LC3-II is considered to be a good marker for monitoring the occurrence of autophagic activity (28). P62, ubiquitin binding protein, is a marker protein that reflects autophagic activity (29). When autophagy occurs, p62 levels are decreased, while autophagic activity is inhibited, p62 protein is accumulated (30). mTOR, the mechanistic target of rapamycin, functions as a sensor that respond to many metabolic events to regulate cell growth and homeostasis including the control of autophagy. mTOR exists in the form of two complexes, which are rapamycin sensitive $\mathrm{mTORC} 1$ and rapamycin resistant mTORC2 (31). Numerous studies indicate that miRNAs play significant roles in cancer development by acting on mTOR itself, mTOR pathway or the key factors within the mTOR pathway.

Rictor, as the crucial component of mTORC2, plays an essential role in regulating and activating Akt phosphorylation (32). It has been reported that activated Akt further regulates cell growth, apoptosis and autophagy by triggering the phosphorylation activation of mTOR, including GC $(21,33,34)$. The Akt-mTOR signaling pathway is a dominant negative signaling pathway that is resistant to autophagy (35). In addition, a preliminary study disclosed that Akt-mTOR activation was positively correlated with Rictor in CNE and HeLa cells (21). In line with previous studies, we provided evidence that Rictor rescued miR-let-7a-induced autophagic activity, which suggested a direct link between miR-let-7a and Rictor. Moreover, the phosphorylation of Akt and mTOR could be damaged by upregulation of miR-let-7a and be strengthened by downregulation of miR-let-7a. These results show that miR-let-7a regulates autophagy by directly targeting Rictor via Akt-mTOR signaling pathway in GC cells. 
There is no denying that the present study has certain limitations. Our research was incomplete and limited, what we discovered cannot represent GC. We only verified the hypothesis in gastric cell lines MGC-803 and SGC-7901, other gastric cell lines, such as BGC-823, AGS and so on, were not included in the research. Our data showed that miR-let-7a promote autophagy through Rictor/Akt-mTOR pathway, however, the interaction between cytokines in cancer cells is very complex, we cannot rule out other signal pathways affected by miR-let-7a which may influence Akt-mTOR expression.

Taken together, the present study demonstrated that miR-let-7a works as a promoter at aspect of autophagy in GC cells, and Rictor is its direct target gene. In addition, we revealed that miR-let-7a may play its role in promoting autophagy through Rictor/Akt-mTOR pathway. To the best of our knowledge, that miR-let-7a targets Rictor has never been reported in GC cells. Therefore, targeting miR-let-7a/Rictor/autophagy pathway in clinical treatment of GC requires further study and exploration.

\section{Acknowledgements}

The present study was funded by the Natural Science Foundation of Jiangsu Province [grant no. BK20131447 (DA13)], the 'Medical ZhongDianRenCai Project' of Jiangsu Province (grant no. RC2011059), and the '333 Project' of Jiangsu Province [grant no. BRA2013280 (RS13)].

\section{References}

1. Ferlay J, Soerjomataram I, Dikshit R, Eser S, Mathers C, Rebelo M, Parkin DM, Forman D and Bray F: Cancer incidence and mortality worldwide: Sources, methods and major patterns in GLOBOCAN 2012. Int J Cancer 136: E359-E386, 2015.

2. Yang L, Parkin DM, Ferlay J, Li L and Chen Y: Estimates of cancer incidence in China for 2000 and projections for 2005 Cancer Epidemiol Biomarkers Prev 14: 243-250, 2005.

3. de Martel C, Forman D and Plummer M: Gastric cancer: Epidemiology and risk factors. Gastroenterol Clin North Am 42: 219-240, 2013.

4. Song IS, Oh NS, Kim HT, Ha GH, Jeong SY, Kim JM, Kim DI, Yoo HS, Kim CH and Kim NS: Human ZNF312b promotes the progression of gastric cancer by transcriptional activation of the K-ras gene. Cancer Res 69: 3131-3139, 2009.

5. Pietrocola F, Izzo V, Niso-Santano M, Vacchelli E, Galluzzi L, Maiuri MC and Kroemer G: Regulation of autophagy by stress-responsive transcription factors. Semin Cancer Biol 23 310-322, 2013.

6. Levine B and Klionsky DJ: Development by self-digestion: Molecular mechanisms and biological functions of autophagy. Dev Cell 6: 463-477, 2004.

7. He C and Klionsky DJ: Regulation mechanisms and signaling pathways of autophagy. Annu Rev Genet 43: 67-93, 2009.

8. Liang C and Jung JU: Autophagy genes as tumor suppressors. Curr Opin Cell Biol 22: 226-233, 2010.

9. Moreau K, Luo S and Rubinsztein DC: Cytoprotective roles for autophagy. Curr Opin Cell Biol 22: 206-211, 2010.

10. Wang MC, Wu AG, Huang YZ, Shao GL, Ji SF, Wang RW, Yuan HJ, Fan XL, Zheng LH and Jiao QL: Autophagic regulation of cell growth by altered expression of Beclin 1 in triple-negative breast cancer. Int J Clin Exp Med 8: 7049-7058, 2015.

11. Sui H, Shi C, Yan Z and Li H: Combination of erlotinib and a PARP inhibitor inhibits growth of A2780 tumor xenografts due to increased autophagy. Drug Des Devel Ther 9: 3183-3190, 2015.

12. Tanzer A and Stadler PF: Molecular evolution of a microRNA cluster. J Mol Biol 339: 327-335, 2004.

13. Selbach M, Schwanhäusser B, Thierfelder N, Fang Z, Khanin R and Rajewsky N: Widespread changes in protein synthesis induced by microRNAs. Nature 455: 58-63, 2008.
14. Frankel LB, Wen J,Lees M, Høyer-Hansen M,Farkas T, Krogh A, Jäättelä M and Lund AH: microRNA-101 is a potent inhibitor of autophagy. EMBO J 30: 4628-4641, 2011.

15. Brest P, Lapaquette P, Souidi M, Lebrigand K, Cesaro A, Vouret-Craviari V, Mari B, Barbry P, Mosnier JF, Hébuterne X, et al: A synonymous variant in IRGM alters a binding site for miR-196 and causes deregulation of IRGM-dependent xenophagy in Crohn's disease. Nat Genet 43: 242-245, 2011.

16. Kovaleva V, Mora R, Park YJ, Plass C, Chiramel AI, Bartenschlager R, Döhner H, Stilgenbauer S, Pscherer A, Lichter P, et al: miRNA-130a targets $A T G 2 B$ and DICERl to inhibit autophagy and trigger killing of chronic lymphocytic leukemia cells. Cancer Res 72: 1763-1772, 2012.

17. Tang R, Yang C, Ma X, Wang Y, Luo D, Huang C, Xu Z, Liu P and Yang L: MiR-let-7a inhibits cell proliferation, migration, and invasion by down-regulating PKM2 in gastric cancer. Oncotarget 7: 5972-5984, 2016.

18. Sarbassov DD, Ali SM, Kim DH, Guertin DA, Latek RR, Erdjument-Bromage H, Tempst P and Sabatini DM: Rictor, a novel binding partner of mTOR, defines a rapamycin-insensitive and raptor-independent pathway that regulates the cytoskeleton. Curr Biol 14: 1296-1302, 2004.

19. Laplante M and Sabatini DM: mTOR signaling in growth control and disease. Cell 149: 274-293, 2012.

20. Huang N, Wu J, Qiu W, Lyu Q, He J, Xie W, Xu N and Zhang Y: MiR-15a and miR-16 induce autophagy and enhance chemosensitivity of Camptothecin. Cancer Biol Ther 16: 941-948, 2015.

21. Wan G, Xie W, Liu Z, Xu W, Lao Y, Huang N, Cui K, Liao M, He J, Jiang Y, et al: Hypoxia-induced MIR155 is a potent autophagy inducer by targeting multiple players in the MTOR pathway. Autophagy 10: 70-79, 2014.

22. Liu K, Huang J, Xie M, Yu Y, Zhu S, Kang R, Cao L, Tang D and Duan X: MIR 34A regulates autophagy and apoptosis by targeting $H M G B 1$ in the retinoblastoma cell. Autophagy 10: 442-452,2014.

23. Korkmaz G, le Sage C, Tekirdag KA, Agami R and Gozuacik D: miR-376b controls starvation and mTOR inhibition-related autophagy by targeting ATG4C and BECN1. Autophagy 8: 165-176, 2012

24. Faller WJ, Jackson TJ, Knight JR, Ridgway RA, Jamieson T, Karim SA, Jones C, Radulescu S, Huels DJ, Myant KB, et al: mTORC1-mediated translational elongation limits intestinal tumour initiation and growth. Nature 517: 497-500, 2015.

25. Xu J, Wang Y, Tan X and Jing H: MicroRNAs in autophagy and their emerging roles in crosstalk with apoptosis. Autophagy 8: $873-882,2012$.

26. Mazumder A, Bose M, Chakraborty A, Chakrabarti S and Bhattacharyya SN: A transient reversal of miRNA-mediated repression controls macrophage activation. EMBO Rep 14: 1008-1016, 2013.

27. Su Z, Yang Z, Xu Y, Chen Y and Yu Q: MicroRNAs in apoptosis, autophagy and necroptosis. Oncotarget 6: 8474-8490, 2015.

28. Kimura S, Fujita N, Noda T and Yoshimori T: Monitoring autophagy in mammalian cultured cells through the dynamics of LC3. Methods Enzymol 452: 1-12, 2009.

29. Klionsky DJ, Abeliovich H, Agostinis P, Agrawal DK, Aliev G, Askew DS, Baba M, Baehrecke EH, Bahr BA, Ballabio A, et al: Guidelines for the use and interpretation of assays for monitoring autophagy in higher eukaryotes. Autophagy 4: 151-175, 2008.

30. Mathew R, Karp CM, Beaudoin B, Vuong N, Chen G, Chen HY, Bray K, Reddy A, Bhanot G, Gelinas C, et al: Autophagy suppresses tumorigenesis through elimination of p62. Cell 137: 1062-1075, 2009.

31. Jung CH, Ro SH, Cao J, Otto NM and Kim DH: mTOR regulation of autophagy. FEBS Lett 584: 1287-1295, 2010.

32. Sarbassov DD, Guertin DA, Ali SM and Sabatini DM: Phosphorylation and regulation of Akt/PKB by the rictor-mTOR complex. Science 307: 1098-1101, 2005.

33. Liu Y, Sun Y and Zhao A: MicroRNA-134 suppresses cell proliferation in gastric cancer cells via targeting of GOLPH3. Oncol Rep 37: 2441-2448, 2017.

34. Ying J, Xu Q, Liu B, Zhang G, Chen L and Pan H: The expression of the PI3K/AKT/mTOR pathway in gastric cancer and its role in gastric cancer prognosis. Onco Targets Ther 8: 2427-2433, 2015.

35. Shintani T and Klionsky DJ: Autophagy in health and disease: A double-edged sword. Science 306: 990-995, 2004. 